\title{
Pour une théorie de la communication en contexte de travail appuyée sur des théories de l'action et de l'expression
}

Pierre Delcambre

\section{(2) OpenEdition Journals}

Édition électronique

URL : http://journals.openedition.org/communicationorganisation/108

DOI : 10.4000/communicationorganisation. 108

ISSN : 1775-3546

Éditeur

Presses universitaires de Bordeaux

Édition imprimée

Date de publication : 1 juillet 2007

Pagination : $42-63$

ISSN : 1168-5549

\section{Référence électronique}

Pierre Delcambre, « Pour une théorie de la communication en contexte de travail appuyée sur des théories de l'action et de l'expression », Communication et organisation [En ligne], 31 | 2007, mis en ligne le 01 juillet 2010, consulté le 02 mai 2019. URL : http://journals.openedition.org/

communicationorganisation/108; DOI : 10.4000/communicationorganisation.108 
Dossier : Migrations conceptuelles. D'où viennent les concepts de la communication organisationnelle?

\section{Résumé}

En contexte de travail, dans les échanges au sein des organisations ou entre organisations, les conditions de l'activité communicationnelle des acteurs sociaux, des salariés, ne relèvent que difficilement d'une théorie de l'expression publique. L'article vise à proposer quelques principes d'une description des échanges, dans les organisations, entre organisations, qui tentent d'être cohérents, dans une approche socioanthropologique de la communication, avec les théories de l'action et les théories de l'énonciation.

Mots-clés : Formes communicationnelles, genres, place, sujet, énonciation, actes de langage, activité collective.

Abstract In the work environment, where there is interchange both in and between organizations, it may be difficult to construct a theory of free and public expression in relation to communication analysis.This paper aims to describe five possible parameters for language exchange between the groups concerned. The methods used are social-anthropology and action and expression theory. First we suggest a need to re-evaluate established forms of communication which are well known in the work place and, secondly, evaluate the commitment behind each expression. Thirdly, stress the necessity of undertaking a detailed analysis of the potentially shifting standpoints in the discourse between the addressor and the addressee. Fourth, identify the "when" of discussion and written communication in the potential development of working strategies between the salaried staff and the managerial structure and, therefore, treat communication as a distinct activity in itself.

Keywords : Forms of communication, place, subject, enunciation, expression, speech acts, group activity.

Pierre Delcambre est Professeur en SIC à l'Université Lille 3, et membre de l'équipe «Communications Organisationnelles et Processus d'Innovation »-COPI- de Geriico. Il développe une analyse des pratiques et activités de communication ordinaires (communications de travail, communications publiques) d'entreprises et d'organisations (Travail social, Chantiers navals, Marine marchande, secteur culturel du spectacle vivant). Il cherche à définir les formes stables des échanges ainsi que leurs dynamiques. 


\section{Pour une théorie de la communication en contexte de travail appuyée sur des théories de l'action et de l'expression Pierre Delcambre}

pierre.delcambre@univ-lille3.fr

\section{Pour introduire en s'inscrivant dans un champ de questions...}

Quand on souhaite que «la Communication organisationnelle se constitue en cadre d'analyse dont l'ambition est de mieux comprendre les organisations à partir des phénomènes de communication qui les traversent $\gg \ldots$

"L'étude de la communication des organisations s'est étendue à l'observation des phénomènes communicationnels au sein des organisations... » disait l'appel à communication de ce numéro de Communication \& Organisation. J'ai longtemps défendu une telle position, intitulant régulièrement mon séminaire de recherche « communications des organisations, communication dans les organisations", pour ouvrir à mes propres recherches sur les « communications de travail dans les organisations ». C'était alors une manière de vouloir que notre champ scientifique se démarque d'une définition par la formation aux métiers de la communication, champ d'études obligé pour des enseignants chercheurs le plus souvent en poste dans des formations professionnelles de «communication », ou d'une définition par les acteurs majeurs du champ, les divers « communicants d'entreprise ». C'était aussi une manière de faire entrer le champ des études de Langage et Travail dans les «communications organisationnelles" et les Sciences de l'Information et de la Communication. Mais cela devient aussi une nécessité dès lors que nos terrains ne sont plus forcément des «entreprises »: la notion même d'organisation, utilisée jusque là simplement comme générique subsumant les organisations que seraient des entreprises de process, des multinationales, des PME de services, des associations gestionnaires ayant des missions publiques, des associations caritatives... la liste des terrains est longue... change radicalement de sens dès lors que l'on étudie les relations structurées d'échange entre l'entreprise et ses sous-traitants, entre l'entreprise et ses consultants, entre l'entreprise et ses actionnaires, ses "parties prenantes», ou encore le jeu en réseau de «partenaires » dont l'action en réseau est pilotée ou non ( réseaux » ou associations tels ceux que 
Dossier : Migrations conceptuelles. D'où viennent les concepts de la communication organisationnelle?

l'on trouve ordinairement dans le secteur culturel, en France). On n'est même plus sûr que l'on puisse, dans ces cas là, dire " au sein des organisations » : car « au sein de » fait signe pour d'autres réalités et " organisation » prend alors un autre sens. Cette fois,-ci il identifierait les états de relation qui visent à conforter les relations d'échange. Le texte qui suit voudrait précisément indiquer quelques pistes et enjeux d'un travail de recherche qui estime que notre discipline se doit aussi de décrire, si possible avec une base théorique commune, les états des échanges, donc les formes de ces échanges, qui sont stabilisés comme moments de communication et seront ou non décrits par les acteurs comme une « organisation ».

\section{Quelques éléments du cadre de référence}

Il suffira de dire, pour que le lecteur situe mon univers de recherche, que la plupart de mes travaux concernent l'analyse des jeux entre la définition hiérarchique des cadres de l'activité et la mise en œuvre de cette activité, sous des formes de coopération issues d'organisations fortement structurées par des «métiers ». Cette perspective d'analyse convenait aux terrains qui ont été les miens (Education spécialisée et travail social, Chantiers navals, Marine marchande, Etablissements de production et de diffusion du Spectacle Vivant).

Mon ancrage théorique est fait :

1. d'une anthropologie de la communication (ce sont des hommes, pris dans des collectifs, qui vivent à leur tour des phénomènes, qui ne sont pas « neufs », mais qui sont pris pour eux dans le temps « actuel» de leur travail, de l'activité collective),

2. d'une définition du champ de la communication comme analyse des relations instituées et faites d'échanges, donc de langage (une attention aux formes langagières, à leur inscription dans la durée, dans des dispositifs, dans des responsabilités où le sujet qui parle est « au travail », encore moins «maître de lui -et de sa parole- comme de l'univers » qu'ailleurs),

3. d'une conception de ce que l'activité qu'est le travail est collective, organisée ; et que comme toute activité collective, elle est faite de sens et de non sens, de projets et de routines, de partage et de rien qu'à moi.

Si j'indique ainsi un cadre global de référence, c'est que, dans cet article, je souhaite d'abord travailler sur la description même des situations appelées "communication». Et travailler ici en termes de cohérence de l'approche théorique. 
Très souvent nos travaux -ainsi que la procédure que nous conseillons à nos étudiants- démarrent avec une description " sociologique » (ou socio-économique) de l'organisation (« artefact» ou « institution»); nous repérons alors des "systèmes d'acteurs"; ensuite, selon les objectifs on cherche à étudier avec plus de précision des «outils et leurs usages », des "dispositifs», des "pratiques de métier», des « nouvelles normes »... Je pense aussi pour ma part que diverses sociologies sont une base analytique de compréhension. Mais pourquoi alors avons-nous des méthodes et des approches différentes des sociologies ? Seulement parce notre "zone d'observation » est plus réduite ? Je pose pour ma part que notre apport, la mise au point de méthodes spécifiques proviennent de la prise en compte que l'on ne peut pas traiter des communications comme de n'importe quelle action collective. Pour commencer, on ne peut exactement les décrire seulement ainsi, sauf à opérer des réductions simplificatrices qui font perdre tout intérêt à être un « chercheur en communication ».

\section{En conséquence, proposer cinq principes d'analyse}

Je voudrais expliciter ici pourquoi, quand je dois employer une formule rapide, je dis que nous devons, pour développer une science de la communication, être aussi forts et informés sur les théories de l'action que sur les théories de l'énonciation (mon propos est de désigner un objectif : je ne prétends pas être déjà cet expert-là !). En présentant ce qui me paraît comme 5 principes d'analyse ouvrant à chaque fois sur des concepts clefs :

Principe 1: on retiendra comme principe descriptif de base d'une approche anthropologique que les phénomènes de communication, les genres sont connus et reconnus par des communautés (notamment des communautés de travail : métiers et autres). Les communications ne peuvent donc être simplement analysées comme des interactions, réalisées hic et nunc. Le cadre (Setting chez Hymes) et les genres sont donc deux concepts descriptifs centraux. Si l'analyse du "cadre » permet de voir comment sont stabilisées des formes, ainsi que des places pour les participants, l'effectuation de la communication demande qu'on fasse appel à une théorie de l'expression.

Principe 2: une théorie de l'expression renvoie à une posture scientifique vis-à-vis de la question du sujet. Celui qui parle est dans du discours (discours circulant); il n'est pas en «maîtrise ». Il n'est pas non plus forcément "adhérent» à ses propres paroles (dans les univers de travail c'est là un phénomène trop récurrent pour que l'on accepte simplement une théorie de l'expression publique comme base 
Dossier : Migrations conceptuelles. D'où viennent les concepts de la communication organisationnelle?

de travail). Et précisément il faut, dans le cadre du travail analyser la posture particulière de celui qui «communique» dans le travail. Le salarié : quel sujet ? Et encore : quel "porte-parole»? A côté d'une théorie de l'engagement (dans l'activité collective) c'est une théorie de l'énonciation et des conditions d'expression dans l'activité collective dont nous avons besoin.

Principe 3 : une théorie conséquente de l'expression exige de traiter vigoureusement la différence entre le destinataire et le récepteur. Le destinataire étant une figure discursive dans l'énoncé, une place imaginaire construite pour le «supposé à la réception » et qui vise à le déplacer. D'où la possibilité de travailler en termes d'action (cible, projet, stratégie). Vouloir déplacer l'autre n'est qu'un projet (« ordinaire » de la relation) non un « fait», une entreprise réussie à tout coup. Cela peut être un élément de la dynamique des échanges, mais ce genre de dynamique (souvent renvoyée à un "agir» individuel) ne nous dit pas dans quelle action communicationnelle s'inscrivent les échanges. Les avancées de la "pragmatique » sur les actes de langage permettront-elles de faire le pont entre le travail d'énonciation du sujet et le cadre réglé d'échanges, qui ne sont pas simplement ajustement et négociation permanente du cadre?

Principe 4 : que les relations soient des pratiques communicationnelles ne nous exonère pas de décrire dans quelle action collective sont pris les « acteurs sociaux » du monde étudié. Si les acteurs sociaux ne sont que rarement de simples «exécutants » ou opérateurs, il ne suffit pas de distinguer travail prescrit et travail réel pour analyser l'activité ; des régimes d'activité (de management de l'activité) induisent des formes pilotées et des réponses communicationnelles des salariés fort diverses. Mais qu'est-ce qui, de l'activité communicationnelle est "managé », quand les salariés participent-ils à la formalisation de l'activité communicationnelle ? Constituer l'ensemble de ce travail en observable n'est pas si simple: l'activité n'est pas que le réel observable, ou plus précisément elle ne se résume pas au temps de la mise en œuvre. La conception est aussi pour certains, dans la division du travail, un temps fondamental. Enfin une perspective d'analyse de l'activité -notamment communicationnelle- ne peut simplement comprendre la contribution expressive des salariés comme une actualisation informée des formes disponibles instituées et donc déjàlà : pour ceux qui sont " engagés » dans l'activité collective, le sens de l'activité collective, le sens de la tâche -étant donnée la division du travail- « oriente » l'action, et « orientant» la relation, peut ouvrir à la proposition de formes communicationnelles inédites dans leur organisation . 
Pour une théorie de la communication en contexte de travail...

Principe 5 : les «moments » d'échange ne sont donc pas analysables seulement comme le proposait le principe anthropologique 1 qui cherchait à repérer les formes stables connues des communautés, ils ne sont pas seulement des rituels et des genres fonctionnant en durée longue: ils sont institués ou laissés informels selon le cadre de l'activité et selon le "management» de l'organisation, ils sont « inventés » en fonction d'opportunités qui font l'histoire située de collectifs.

\section{Pour une approche anthropologique des échanges : le rôle des communautés dans le repérage des formes communicationnelles.}

Je commence par ce point, dans la mesure où j'ai accepté de définir mon ancrage comme «anthropologique». Qu'est-ce que cela signifie? Il y a bien eu une approche anthropologique des mondes contemporains (Althabe, 1992 ; Augé, 1994 ; Fabre, 1993), mais une telle approche est rare pour analyser des «communications organisationnelles " (on trouvera en revanche des références à l'anthropologie du projet de Boutinet: Boutinet, 1990). L'anthropologie cognitive (Goody, en particulier Goody, 1986) est plus régulièrement citée par les analystes de la communication écrite de travail. En fait, c'est le soin que j'estime nécessaire à la description des formes communicationnelles qui me semble exiger une approche de type anthropologique. C'est le parti-pris des "gens » : il n'est de communication que de gens, ce qui conduira à la fois à parler des machines comme " assistance à la communication » et à travailler sur la communication des entreprises (ou des organisations...) comme communication «au nom des entreprises». L'approche anthropologique permet aussi de s'installer dans le temps long, qui permet d'identifier des formes communicationnelles stabilisées. J'ai utilisé le terme de «forme communicationnelle» pour me permettre d'englober ensemble l'analyse de ce que nous nommions dans les années soixante-dix «situations de communication » (travaillées par les «techniques d'expression » de l'époque, voir Vanoye, 1990), et l'analyse des supports et des genres. Dans un tel cadre, nous disposions seulement alors du terme de "contexte» pour ne nous enfermer ni dans l'analyse ponctuelle des échanges, ni dans l'analyse réduite à celle du produit fini. Or une approche anthropologique de la communication comme celle, certes ancienne, de Hymes (Hymes, 1967 présentée dans Bachmann et al, 1981 : 72-76) permet de poser des concepts descriptifs et de les travailler. 
Dossier : Migrations conceptuelles. D'où viennent les concepts de la communication organisationnelle?

A partir du modèle descriptif «SPEAKING $»^{1}$, je propose d'abord de prendre appui sur la description des «genres ». Ce qui m'intéresse dans la perspective anthropologique, c'est que les "genres» (plus largement les "formes communicationnelles ») sont reconnus par la communauté. Cet angle d'attaque est fondamental et précisément ouvre à des questions et difficultés que nous devons résoudre ${ }^{2}$. Une première difficulté est précisément de donner au terme de communauté une consistance, dès lors que nous cherchons à étudier des milieux de travail. Autant certaines formes sont repérées et identifiées dans des communautés restreintes (formes que l'on pourrait dire issues d'une socialisation secondaire pour reprendre Dubar, Dubar, 1991 : 100-107- , telles que les « notes de comportement », ou « une réunion de synthèse » dans l'Education spécialisée -Delcambre, (1997)-, la «pres »-Gaglio et al (2006), autant d'autres participent d'une socialisation primaire (un «rapport» par exemple, une «notice »). En tout cas cette approche incite à commencer ethnométhodologiquement par décrire les formes communicationnelles repérées et leurs appellations. Une histoire de ces formes nous permet aussi de mettre à jour ce qui fait «cadre" pour les participants, et ce qui a été durablement installé comme dispositif (de l'architecture aux moyens techniques) dans lequel s'inscrivent à chaque fois ces formes. Faire un point sur l'usage que nombre de chercheurs en SIC font du concept de «cadre » n'est pas mon propos ; certes « Setting » chez Hymes n'est pas le « Frame» de Goffman (voir Joseph, 1998: 63-69), ni « dispositif»... Ce que j'entends surtout souligner en commençant par ces deux conceptsrepères (genre et cadre), c'est que les participants s'engagent dans des processus de communication déjà structurés, déjà mis en place. Ces formes sont stabilisées, ce qui va amener le chercheur à étudier d'abord ce qui rend l'activité communicationnelle stable, avant d'en

\footnotetext{
${ }^{1}$ Présenté dès 1981 dans l'ouvrage de Bachman, Simonin et Lindenfeld, (ouvrage réédité récemment) le modèle se présente comme un acronyme -utile à la mémorisation- amenant à décrire une situation de communication selon 8 modalités : la description du «cadre » (Setting), des «Participants », des finalités de la situation «Ends», des moments ou «Actes» propres à la situation, des «tonalités expressives » (Keys), des «Instrumentalities » moyens humains et techniques mis en œuvre, des Normes et des Genres (formes repérées par la communauté). Cet outil descriptif date des années 1960.

${ }^{2}$ L'une des questions délicates est précisément celle de l'agir et des «actes de langage ", j'essaierai de revenir sur ce point. Dans le modèle de Hymes "acts » amène à l'analyse dramaturgique, celle de la trame du déroulement de la situation (il ne s'agit donc pas d'une perspective pragmatique). L'analyse dramaturgique a été largement explorée dans le contexte des études conversationnelles (voir encore Licoppe, 2007) ou dans l'analyse de la structure interne des genres et des documents.
} 
étudier les caractères innovants. Cette «stabilité » est précisément à décrire; on peut pour cela emprunter à l'analyse des «rituels" (Goffman encore, mais aussi Augé, -Augé, 1994 : 81-126-, Rivière Rivière, 1995-). Mais la stabilité des formes communicationnelles, étant données les grandes différences entre «entreprises» dans les investissements financiers pour établir des formes communicationnelles (appui à l'interne ou à l'externe sur des spécialistes des formes, de l'édition, sur des organisateurs d'évènements, sur des services protocolaires divers) n'est pas si simplement établie: la diffusion et la progressive installation de formes standard font partie de l'enquête. Il y a ainsi tout un «backoffice » de la production communicationnelle.

Nombre de chercheurs mettent en lumière ce back-office, notamment tous ceux qui analysent les systèmes d'information, l'énonciation éditoriale et les documents (Souchier et al, 2003, Cotte, 2006). En contexte de travail, nombre de formes communicationnelles sont déjà structurées, normées. Parfois elles sont en cours d'installation comme « forme communicationnelle sous organisation $»^{3}$, d'où l'attention que l'on peut porter aux consultants et à tous ceux qui «assistent» (tuteurs, manuels, consignes). Prendre le «parti des gens » amènera à mettre en valeur le corollaire de «l'assistance»: les «gens» qui participent à la production de ces communications apprennent à tenir leur place dans la communication en s'y engageant ; les salariés sont en devoir de s'engager dans des communications pour eux parfois inédites ${ }^{4}$. Ce terme d' "engagement» n'étonnera pas : nous sommes nombreux à nous y référer dès lors que notre angle d'analyse est de chercher à comprendre la communication faite par "les gens », et, dans le cadre des "communications organisationnelles», faite la plupart du temps par des salariés. «Engagement» «mobilisation » (des salariés : Le Goff -Le Goff, 1993-; de la force de travail : De Crescenzo -De Crescenzo, 2005-).

\section{Pour l'expression, quel sujet ?}

On s'étonnera plus que ma démarche anthropologique débouche sur un appel à une «théorie de l'expression». Certes, c'est une décision qui peut se discuter que de réduire «la communication » aux formes langagières et aux échanges langagiers; la microsociologie

\footnotetext{
${ }^{3}$ Voir Basse, 2003, en ce qui concerne les «projets d'établissement» dans le secteur hospitalier.

${ }^{4}$ L'inédit pour un acteur, un énonciateur n'est pas forcément «innovant » dans le cadre plus large des « communautés ».
} 
Dossier : Migrations conceptuelles. D'où viennent les concepts de la communication organisationnelle?

goffmanienne nous a appris tout l'intérêt, dans l'analyse des interactions, de l'observation des déplacements, de l'analyse du visible. Mais dès lors qu'on assume cette réduction du champ d'analyse, nous nous retrouvons avec des productions langagières. L'engagement dans la production implique que nous traitions de l'expression.

Ce point est celui qui pose pour moi le plus de difficultés. Autant nous sommes aidés pour travailler sur l'engagement (d'Elias à Goffman et son « involvment», mais aussi avec J. Ion -Ion, 2001- sur les nouvelles formes d'engagement dans l'espace public, et Sainsaulieu pour l'analyse des formes de démotivation et de retrait dans le travailSainsaulieu, 1993-...), autant nous peinons sur les questions d'expression. Et rares sont les auteurs qui se risquent à ces analyses (parmi les chercheurs français, J. Caune -Caune, 1995: 114-116-, mais aussi en dehors de cet espace de chercheurs, A. Eraly -Eraly, 2000- en Belgique), mais sans chercher à penser les spécificités des univers de travail. Or, à un moment du travail sur «la communication", il faudra bien se confronter à la question du «sujet» (de l'action, de l'énonciation), ne serait-ce que parce que l'analyste se doit de dire «au compte de qui» mettre la communication. Et que la question ne se règle pas simplement en termes d'" auteur», ni même de «pilote de l'action communicationnelle». En fait nous avons du mal à concilier des avancées majeures d'autres disciplines avec une théorie de l'expression pertinente pour nos travaux. Ces avancées majeures nous amènent à ne plus poser comme "sujet de l'expression » un sujet «plein», possesseur de son énoncé, maître de son énonciation, développant sans aléa ni détour la préconception «stratégique » de son intervention langagière dans son propos. Nous avons appris cela et en avons tiré quelques conséquences. Mais cela laisse ouverte la question de la genèse des collectifs, cela laisse ouverte la question de la place et de la responsabilité de l'énonciateur, la question des rapports de domination et d'engagement dans l'activité, si l'on veut aller au-delà de l'analyse des potentialités communicationnelles des outils et des formes instituées. Pour poser quelques points sensibles je me propose de revenir sur les avancées ouvertes par le dialogisme bachtinien, sur la question du porte-parole pour en terminer avec des interrogations sur le salarié comme sujet.

Que le sujet du discours est «traversé » de la parole et des discours des autres, c'est un principe d'analyse qui permet de renouveler l'étude de la production idéologique, du bouclage des énoncés par la propagande. Déjà Flahaut (Flahaut, 1978) avait tenté de faire le pont 
entre production langagière du sujet et pré-existence de discours disponibles, en travaillant sur la réponse au «manque à dire » que représentait la reprise de motifs tout-prêts, repris comme « insignes $»^{5}$. Dans le monde des « communications des organisations » nous avons, bien sûr, à analyser les constructions idéologiques multiples d' « auteurs $»^{6}$. Mais, dès lors que nous ne nous situons plus dans une analyse «en masse » de l'idéologie et qu'il nous faut travailler la « reprise », le « réemploi », voire les décalages produits dans des communications situées ${ }^{7}$, comment travailler l'engagement expressif de salariés qui ne produisent pas des textes «à compte d'auteur»? Comment travailler non seulement l'engagement expressif des salariés de front office en contact avec la clientèle (ce qui avait été régulièrement travaillé par les chercheurs de Langage et Travail) mais aussi celui des cadres et de leurs relations aux autres «managers» du management intermédiaire comme du top management ? Comment travailler, dès lors que le sujet de l'énonciation «n'adhère pas » à son énoncé ? Que le sujet du discours soit «parlé » est une chose ; nous devons quant à nous, en analysant les formes communicationnelles, aller au devant d'autres problèmes qui nous obligent à nous interroger sur « l'expression $»^{8}$.

Une des manières possibles est de travailler sur la construction des " porte-parole ». Un tel travail, s'il s'avère utile et classique dès lors que l'on travaille sur les relations stabilisées entre organisations, est beaucoup plus délicat dès lors que l'on travaille «au sein de l'organisation ». Travailler sur la normalisation de la communication par les chartes graphiques, travailler sur les définition des formats, leur édition, leur mise en circulation, travailler sur la place de l'expression des salariés dans les «supports de communication institutionnels » n'exonère pas de mettre en place une description du

\footnotetext{
${ }^{5}$ Le travail de Ph.Breton (Breton, 1997) sur l'argumentation me semble s'appuyer aussi sur cette existence de discours circulants, comme une conscience discursive du " déjà-là » pour celui qui vise à argumenter.

${ }^{6}$ Tout récemment la thèse de Geneviève Guilhaume (Guilhaume, 2006) tente de travailler sur les corpus des communicants développant le « coaching». Il resterait néanmoins à évaluer, en analystes de discours, les travaux souvent cités de Boltanski et Chiapello sur le «Nouvel esprit du capitalisme ». Une fois la référence-révérence faite, n'en avons-nous rien à dire ?

${ }^{7}$ Les mondes sociaux sont poreux, certes, mais les discours ne se répandent pas par la seule force des énoncés.

${ }^{8}$ Je laisse de côté la question, travaillée en son temps, du «droit à l'expression » des salariés (Mothé-Gautrat, 1986). Je tâche de me concentrer sur les problèmes liés au rapport du salarié énonçant au «sujet de l'énoncé » quand l'énoncé est à produire en contexte de travail, quand le salarié « communique ».
} 
Dossier : Migrations conceptuelles. D'où viennent les concepts de la communication organisationnelle?

«statut» de l'énoncé d'un salarié dans les communications développées dans l'univers du travail. Une des voies possibles, dès lors que l'on s'intéresse aux collectifs de travail, est de décrire les jeux de "représentation» au sens ici de « délégation » entre l'énonciateur et ceux « au nom de qui il parle». Or il semble que les conditions de délégation et d'accréditation sont moins réglées ici que dans le cadre des associations et groupements politiques organisés pour intervenir dans l'espace public'. Le travail qu'avait fait en son temps Bourdieu (Bourdieu, 1982; Bourdieu, 2001) pour critiquer l'analyse Austinienne des actes de langage (Austin, 1970) revenait à vouloir définir le "langage autorisé », notamment en s'intéressant aux signes d'accréditation (le pouvoir symbolique du Skeptron) ${ }^{10}$. La multiplicité des communautés possibles dans l'entreprise, la dissociation progressive des communautés professionnelles anciennes sous le coup d'organisations non durables (de type " projet»), le jeu ordinaire des contradictions de place ou de point de vue -salarié d'une entreprise, salarié d'un site, occupant d'une place, " capitaliste » employable ou ruiné de ses compétences et de son expérience- amènent qui veut traiter des énoncés des salariés à expliquer quelles sont les places d'énonciation disponibles dans les communications ordinaires (non suscitées par l'enquête -Delcambre, 2000). De fait, les situations ou régimes d'énonciation laissent penser que le statut de la parole ou de la production écrite des salariés est variable : le salarié n'est pas à tout coup dans une situation où il faudrait et suffirait d'attendre que sa parole soit légitimée pour s'acquitter de son « devoir d'expression », ou simplement répondre à la sollicitation d'expression, notamment dans les collectifs de travail.

Il n'est pas envisageable ici de développer la variété des cas de figure. Reste néanmoins à dire que se poser la question «Quel sujet peut être le salarié dans sa parole, dans la production collective -orale ou écrite ?» ouvre les questions de la «contribution» des salariés à l'activité de l'entreprise, ainsi que de la reconnaissance de cette contribution personnelle.

\footnotetext{
9 Je voudrai signaler un travail remarquable, trop peu lu par les spécialistes de communication, de J.Guilhaumou (Guilhaumou, 1998), précisément sur les «porteparole de la République » (1789-1792), travaillant à partir d'archives à comprendre les actes de langage que ces «missionnaires patriotes» ont tenté de poser pour accomplir leur « mission ».

${ }^{10}$ Ces analyses m'avaient amené à travailler, dans des collectifs (« équipes ») faits de membres de métiers différents, les rapports de langage, et la construction plus ou moins évidente du «porte-parole»: Delcambre, 1997, chapitre 6 «Rapports de langage et langage autorisé »p. 237-270.
} 


\section{L'expression, comment articuler le sujet... et ses autres}

Je voudrai, à présent, revenir sur un point qui me paraît décisif quand on cherche à accepter de manière cohérente une théorie de l'expression. Je m'attarderai ensuite sur ce qui pose problème: l'articulation entre le sujet et le "cadre», dès lors que l'on veut travailler la dynamique des échanges.

Dans une théorie de l'expression un concept décisif est celui de « destinataire ». Qui n'a pas, dans ses études, appris « le schéma de Jakobson » (Jakobson, 1963) ? Qui n'a pas à un détour d'une histoire des théories de « la communication», entendu ou fait un exposé sur cette analyse fonctionnelle du langage pris dans la communication ? Mais que faire de ce moment canonique ? Je propose qu'on l'analyse bien comme une "théorie de l'expression»: on suit trop souvent Jakobson lui-même dans sa manière de "succéder» à la représentation «machine » de la communication pour l'améliorer sans prendre la mesure d'une rupture fondamentale. Je la situe dans la distinction radicale qu'il nous invite à faire entre le destinataire et le récepteur. $\mathrm{Si}$, par récepteur, nous entendons non tant les machines que les gens qui sont «à la réception », les « récepteurs » désignent alors des «récepteurs empiriques». Le destinataire est d'une tout autre nature : il est à proprement parler un «construit» du destinateur par son message, une figure imaginaire. C'est bien pour cette raison que ce pôle ouvre à l'analyse d'une fonction « conative » et que nombre d'analystes ont emboîté le pas du destinataire pour travailler sur la « cible ». Or, si nous nous situons dans une perspective d'analyse dynamique (non le message, non l'oeuvre poétique comme message, comme dans le travail de Jakobson) on peut retenir que construire un destinataire, c'est avoir un projet de déplacement de l'autre. C'est lui proposer, dans le message, une "place", des figures d'identification. C'est proposer à cet autre -dans le cours d'action expressive-, telle ou telle figure possible, parmi toutes les possibilités identitaires qui permettent à cet autre de se «tenir» comme sujet dans l'action ${ }^{11}$ et dans les interactions. Le destinataire est donc une figure discursive de l'énoncé, une place imaginaire construite pour le «supposé à la réception » et qui vise à le déplacer. D'où la possibilité de travailler en termes d'action. Mais avec un cadre d'action qu'il faut penser pour

\footnotetext{
${ }^{11}$ Ce contexte identitaire est largement hors du champ de la connaissance que peut avoir le destinateur sur les supposés récepteurs empiriques, sauf à s'informer préalablement (travail classique dans une perspective stratégique) ou à avoir acquis une expérience de l'autre par des échanges réguliers. Mais la connaissance des récepteurs ne réduit pas pour autant le travail du destinateur dans la construction du destinataire.
} 
Dossier : Migrations conceptuelles. D'où viennent les concepts de la communication organisationnelle?

qu'il ne nous piège pas dans l'analyse : celui d'un projet sur l'autre, d'un « coup » dans des jeux supposés stratégiques. Or autant l'analyse du destinataire permet de travailler la dynamique des échanges, autant il est difficile de prendre le "projet sur l'autre » comme un cadre permanent des interactions et des communications collectives. Si Jakobson permet de travailler les rapports de force symboliques qui se jouent au long des échanges, en ce que par le travail sur le destinataire, les locuteurs sont engagés dans des positionnements de soi et des déplacements de l'autre -et de soi-, ces jeux restent pris dans une structuration des échanges. Il s'agit de tenter une analyse qui, au-delà de l'analyse des « coups » et des « tours » de parole dans les interactions, soit en phase avec les enjeux de l'activité collective qu'est le travail.

Une analyse pragmatique des actes (de production et de communication) dans lesquels sont engagés les acteurs nous fournirat-elle un tel cadre ? Je voudrai rapidement explorer les difficultés que nous rencontrons dès lors que nous tentons de penser ces échanges collectifs en nous appuyant sur le terme d' « agir ».

En commençant par dire qu'un mésusage d'Habermas pose une première difficulté. Il me semble important de rappeler que les différents «agir» correspondent à une lecture que fait Habermas d'une littérature scientifique large et qu'il s'agit-là d'une tentative pour clarifier la manière dont ces différentes théories construisent leur appareil théorique d'analyse des rationalités des échanges. Ces différents «agirs» sont donc autant de formes de rationalité des échanges construites et/ou présupposées par des théories et fondées sur des rationalités différenciées propres à différents "agirs de l'acteur ». Or il faudrait retravailler le texte d'Habermas et envisager quand ces rationalités décrites renvoient à l'acteur et quand elles renvoient aux échanges eux-mêmes.

Confondre la rationalité des échanges avec la rationalité des acteurs n'est-ce pas parfois accorder à l'acteur trop de capacité stratégique, parfois l'inscrire dans une simple domination sociale ? Et dans tous les cas n'est-ce pas aussi fermer bien vite la question du « sujet », de son emprise sur la dynamique des échanges, de la consistance de son « je » dans les échanges, que conclure que la rationalité des échanges n'est que la résultante des rationalités des acteurs (la domination d'un acteur dominant, le compromis des négociations gagnant-gagnant, le résultat d'un perdu pour perdu...).

A quel type d'agir référer les logiques d'échanges entre acteurs, si l'on s'appuie un instant sur les quatre « agirs » distingués par Habermas ? 
Le monde de l'entreprise est-il forcément stratégique? La communication de travail est-elle malgré tout intercompréhensive donc « communicationnelle » ? ${ }^{12}$ L'activité ordinaire des salariés estelle fondamentalement «régulée par les normes», et -pour autant qu'elle soit «expressive» l'activité communicationnelle des salariés et des représentants d'organisations est-elle structurellement « dramaturgique », c'est-à-dire orientée par la rationalité qu'Habermas décrit ainsi : " en manifestant quelque chose de sa subjectivité, l'acteur souhaiterait être vu et accepté d'une certaine manière par le public » (Habermas, 1987, t1 : 106) ?

En résumé, l'analyse par la rationalité de l'agir des acteurs ne nous éloigne-t-elle pas de l'analyse des cadres stabilisés des formes d'échange ? Si ceux-ci existent, comment dès lors relier le travail d'expression, ses dynamiques, et l'activité collective, et, à tout le moins « le cadre social de l'action $»^{13}$.

Il me semble que c'est en travaillant plus avant sur «les actes de langage » que nous pourrons faire une proposition théorique cohérente complémentaire à l'analyse des formes communicationnelles stabilisées et reconnues par les communautés. La difficulté actuelle est, me semble-t-il, d'articuler des niveaux d'études différents pour

\footnotetext{
${ }^{12}$ Je me contente ici de faire écho aux discussions régulières de la position de Zarifian (Zarifian, 1996), non pour prendre ma place dans la discussion, mais pour montrer que ces discussions renvoient à une appréciation différente de la consistance des cadres communicationnels (la discussion et ses formes organisées dans l'activité des industries de process) et de la dynamique individuelle, mais partagée des échanges (le projet sinon collectif du moins partagé d'intercompréhension lors de l'échange)

13 J'utilise «collectif» depuis le début de ce texte. Il pose difficulté et je voudrais tenter de m'en expliquer : en ayant à réfléchir sur les communications de travail, j'ai proposé d'abord que, dans une perspective anthropologique, on renvoie l'identification des «formes communicationnelles» à des "communautés ». C'est une manière de travailler effectivement l'identité des formes et leur reconnaissance, mais pas la dynamique des échanges. Dans le même temps, j'ai indiqué que les gens qui communiquent « en contexte de travail » sont pris dans une « activité collective ». Ce terme renvoie à une description de l'action, et plus précisément à la place de celui qui travaille dans une "organisation». J'ai voulu encore chercher une approche théorique capable à la fois d'analyser les échanges entre «membres d'une organisation » et les échanges entre membres d'organisations différentes. Dès lors, les formes de communication permettent de dissocier temporairement certains « membres » de leur organisation -de leur activité collective- pour participer à des processus communicationnels. Ces processus communicationnels sont eux-mêmes faits d'une pluralité d'énonciateurs. Le retour sur une théorie de l'expression s'accompagne chez moi de la nécessité de penser et décrire ces processus d'échanges, sans les réduire à une succession d'expressions individuelles et d'arrangements, et dès lors je suis amené à réutiliser le terme «collectif», les formes communicationnelles étant co-agies.
} 
Dossier : Migrations conceptuelles. D'où viennent les concepts de la communication organisationnelle?

des «actes de langage». Dans l'étendue de ce qui pourraient être autant d'actes de langage, si on ne réduit pas la catégorie aux "performatifs ", on trouve tout aussi bien des analyses du «promettre » (qui sert à travailler le «projet» en particulier) que l'analyse d'actes comme signer (Fraenkel, 1992), nommer, mais aussi faire un bilan (Fauré, 2006), mais encore "rapporter », " négocier », « se concerter» (Bratosin, 2001)... Cette grande diversité n'invalide pas le projet pragmatique à mes yeux, mais exige de travailler à une clarification.

En effet tous les actes de langage n'ouvrent pas de la même manière à la description de ce qui fait cadre pour les énonciateurs. J'ai ainsi pu montrer combien l'acte d'《affirmer» était fondamental pour comprendre les rapports de mer et le journal de passerelle dans la marine marchande (Delcambre, 2002). Au-delà de l'usage ordinaire $\mathrm{du}$ verbe, un usage spécifique existe, qui règle juridiquement les relations et conséquemment le travail d'expression. Affirmer un « rapport de mer » pour un commandant de navire revient à engager la responsabilité de ses propos (le rapport de mer déposé devant l'instance juridique appropriée en cas d'incident en mer) sans avoir le droit de se retrancher, mais avec le droit d'expanser (un complément de rapport devant dans ces cas là être fourni sous durée réglementée elle-aussi). Dans un tel cadre -un dispositif juridique- la charge de la preuve passe au contradicteur. Par cette description lapidaire, je vise surtout à montrer que, dans ce cas, "affirmer" est un acte lié à une institution organisant de manière normative les échanges. L'identification des actes de langage et la compréhension de leur congruence avec des cadres expressifs partagés de l'action communicationnelle fait partie de l'expérience des locuteurs et demande une enquête spécifique à un chercheur " en communication». S'en tenir à l'analyse des «règles de communication » accompagnant l'action serait dommage. D'un point de vue descriptif, nous ne pouvons, non plus, nous en tenir à une description du «statut» des énoncés (comme confidentiel, secret, à diffusion restreinte, publicisé, médiatisé...) : ces concepts descriptifs sont insuffisants pour étudier la dynamique des échanges, comme pour étudier la "création » d'espaces de discussion ou d'autres formes communicationnelles dans les organisations et entre organisations (Huët et Delcambre, 2007).

\section{Les échanges, le cadre d'activité et les dynamiques}

Somme toute, un double principe gouverne l'analyse: inscrire l'analyse des communications dans l'analyse de l'activité ; considérer 
la communication comme une activité elle-même. Inscrire l'analyse des communications dans l'activité, c'est ce que bon nombre d'entre nous faisons. Cela nous amène à poser que toute l'activité n'est pas « communication»: dès lors que nous n'étudions plus l'activité de « services de communication » mais que nous nous intéressons à celle de tous les salariés, nous sommes attentifs aux "moments" où interviennent ces échanges, comme aux individus qui participent -sont exclus- de ces échanges, la participation aux communications «déplaçant» souvent les salariés, tant dans l'espace que symboliquement (dès lors que le «métier» n'est pas de « communiquer »).

Un autre aspect de l'analyse de l'activité nous a amenés à identifier le «travail prescrit» et le «travail réel». Ce jeu de concepts nous a aidés à repérer les différentes manières qui déterminent les formes du travail et pour chacun et dans la production collective. Ici encore les chercheurs ont pu explorer des régimes très divers. Des univers gouvernés par une procéduralisation complète de l'activité -comme dans les lieux de travail «sous qualité", où les jeux de communication peuvent varier $\mathrm{du}$ 《marquage $»^{14}$-il s'agit de « remplir les formulaires » permettant la traçabilité-, à la mise en œuvre de « réseaux clandestins de salariés » (De Crescenzo, 2005)- ; mais aussi des univers de travail où le cadre de l'activité est la mise en œuvre autonome de compétences qui ne sont contrôlées qu'en termes de résultats; ou encore d'autres univers de travail où n'est installé qu'un régime de contrôle faible mais un cadre normé de l'activité collective, où le salarié sait ce qu'il est "censé » faire (Delcambre, 1997); ou encore d'autres, marqués par de nouvelles normes communicationnelles, celles d'un "régime de disponibilité » qui remplacerait un "régime de contrôle» (Pène, 2005). Précisément c'est la diversité des cadres ou des « régimes communicationnels » qui est notable.

Si je devais insister sur un point, c'est sur la proposition de décrire « la communication» elle-même comme une activité, complexe à analyser dès lors qu'elle n'est plus référée à «l'entreprise » ou à un « service ». C'est en faisant le point sur notre vocabulaire descriptif que nous pourrons, me semble-t-il, avancer dans le travail « sous une

\footnotetext{
${ }^{14}$ Je reprends une fois encore la distinction proposée par Charrasse (Charrasse, 1995) qui proposait de différencier trois «régimes d'écriture » : le marquage, la mise en mots et la formalisation. Proposition qui avait l'avantage de permettre aussi l'analyse de la « division du travail des écritures »...
} 
Dossier : Migrations conceptuelles. D'où viennent les concepts de la communication organisationnelle?

théorie de l'action » et le travail "sous une théorie de l'expression» ${ }^{15}$. Pour prendre un exemple, dès lors que notre perspective est "communicationnelle", nous ne pouvons plus nous satisfaire de verbes comme « écrire » (Delcambre, 1997, chapitre 2). Ecrire est trop souvent réduit à « rédiger» (la mise en mot), et on perdrait le cadre collectif de l'action si on oubliait qu'écrire est un temps de l'action collective, un temps qui oriente l'action collective et qui déclenche nombre d'activités : chercher des documents archivés, les multiplier, les relire, se réunir ou discuter par mail interne, penser destinataire et diffusion, proposer une version «martyr», stabiliser des énoncés, emprunter énoncés ou formes diverses, choisir des supports, présenter un état en réunion, discuter, valider, faire signer, définir le cadre de publicisation...

Tout cela n'est pas original et fait bien partie du programme de description que nombre de chercheurs en communication se donnent. Mais cela conduit à penser que toute activité communicationnelle n'est pas facilement observable, et cela explique les nombreuses contributions de collègues quant aux conditions de l'observation d'une part et d'autre part quant à l'importance de la participation à la production des formes communicationnelles elles-mêmes (par exemple la mise au point du développement maison de tel ou tel logiciel, ce qui reviendrait en référence à Hymes, au travail de coconception des « instrumentalities »).

Dernière remarque liée à ce principe d'analyse de l'activité : l'invitation à travailler sur la contribution des salariés à l'activité («travail réel » cité plus haut) nous amène aussi à envisager les ajustements, certes, mais aussi le « sens » que mettent les salariés dans l'activité collective. En d'autres termes, on peut difficilement prendre appui sur une théorie du cadre comme structure déterminant les échanges. Dans les espaces de travail, il est difficile de nier l'importance de la domination, et il est tout autant difficile, anthropologiquement, de poser que les cadres des échanges sont à tout coup inventés par une co-construction. C'est pourquoi il me semble que nous avons tout intérêt à nous appuyer sur les perspectives théoriques, comme la sociologie de l'association de Laville et Sainsaulieu (Laville et Sainsaulieu, 1997), qui permettent notamment

\footnotetext{
15 J'ai bien conscience de l'hésitation que manifeste mon texte, en parlant tantôt de « théorie de l'expression », tantôt de « théorie de l'énonciation ». C'est que la seconde a été et est encore travaillée, alors que la première, qui permettrait de ne pas réduire l'expression à l'énonciation n'est travaillée en tant que, telle que de manière embryonnaire.
} 
Pour une théorie de la communication en contexte de travail...

de penser hors temps long des institutions et des formes communicationnelles instituées: si on a souvent noté que les entreprises et organisations veulent "dés-instituer» des formes de relation, déstabiliser les communautés ou collectifs, on peut tout aussi bien observer que des salariés mettent en place des formes « momentanées » d'échanges, pour autant qu'elles ont du sens pour eux. Le temps des salariés est aussi celui où l'on voit se succéder des «modes communicationnelles », où l'on baisse les épaules pour laisser passer les trains des «nouvelles organisations », où l'on peut...

\section{5 : les formes stables... et les autres}

Voilà pourquoi, s'il est de bonne pratique de commencer par observer les formes stabilisées de communication (principe 1), on peut proposer comme principe complémentaire que les "moments » d'échange ne sont donc pas analysables seulement comme le proposait le principe anthropologique 1 qui s'appuyait sur les formes communicationnelles stabilisées, reconnues par les communautés de travail : elles ne sont pas seulement des rituels et des genres reconnus par la communauté en durée longue; elles peuvent être développées ou non selon le cadre de l'activité et selon le «management» de l'organisation, elles peuvent être «inventées» en fonction d'opportunités qui font l'histoire située de collectifs.

Ce principe est donc d'une autre nature que les précédents. Il n'induit pas une approche théorique nouvelle (le principe d'analyse située de l'activité, y compris l'activité communicationnelle posé en 4 suffit). Il propose plutôt une dynamique de la méthode. Parti d'une analyse des formes stables -conséquente avec le principe 1-, posant que les formes communicationnelles n'existent que mises en œuvre, donc donnant lieu à un engagement expressif de ceux qui, dans l'activité, communiquent (principe 2), acceptant l'idée que l'expression communicationnelle est une action en tant que projet sur l'autre, sans pour autant poser que toute action communicationnelle soit expression maîtrisée et stratégique (principe 3) nous avons insisté sur les formes de l'activité communicationnelle. Cette dernière n'est qu'en partie observable: comme toute activité elle a son "back office», son organisation d'assistance, son pilotage parfois mobilisé, parfois dormant, celui qui se commet à la communication étant lui-même plus ou moins engagé dans l'effectuation, la réflexion et l'investissement de forme.

Ce dernier principe consacre l'importance de l'analyse située (historique et locale), en rappelant que les formes communicationnelles n'ont pas la consistance définitive d'institutions, 
Dossier : Migrations conceptuelles. D'où viennent les concepts de la communication organisationnelle?

qu'elles ne s'imposent pas à tout coup par la domination des agents institutionnels $\mathrm{du}$ changement communicationnel, qu'elles ne stabilisent pas forcément les ajustements et convenances locales des salariés engagés dans l'activité. Cela inscrit notre travail dans une contemporanéité située, que l'observation se fasse in situ (Pène 2001, et tant d'autres) ou résulte d'un travail sur les traces archivées (Delcambre, 1998). Toutes ces études, dont le développement est notable ces dix dernières années, nous donneront la possibilité de comprendre dans quelles conditions des formes organisées de communication peuvent se généraliser, et dans quels contextes elles ne prennent pas effet ou ne perdurent pas.

\section{Conclure ?}

Je pense que le lecteur aura, au cours de ces pages, compris le statut de ce texte: quelques postulats, une ligne directrice, m'ont incité à donner à voir les points qui sont difficiles et demandent de poursuivre le travail de chercheur en communication.

\section{Bibliographie}

Althabe, G., Fabre, D., Lenclud, G., Vers une ethonologie du present, Paris, Ed MSH, 1992.

Augé, M., Pour une anthropologie des mondes contemporains, Paris, Aubier, 1994.

Austin, J.L., Quand dire, c'est faire, Paris, Seuil, 1970.

Bachman, Ch., Lindenfeld, J. Simonin, J., Langage et communications sociales, Paris, Dunod, 1981.

Basse, P., Projet d'Etablissement, cadre de santé et communication de travail à l'Hôpital, Thèse, Université Lille 3, 2003.

Bourdieu, P., Ce que parler veut dire. L'économie des échanges linguistiques, Paris, Fayard, 1982.

Bourdieu, P., Langage et pouvoir symbolique, Paris, Seuil, 2001.

Boutinet, J-P., Anthropologie du projet, Paris, PUF, 1990.

Bratosin, St., La concertation : forme symbolique de l'action collective, Paris, L'Harmattan, 2001.

Breton, Ph, La parole manipulée, Paris, La Découverte, 1997.

Caune, J., Culture et Communication, Grenoble, PUG, 1995. 
Pour une théorie de la communication en contexte de travail...

Charrasse, D., « Marquage et entreprise scripturaire : la construction d'un monde sans auteur », Etudes de Communication, $n^{\circ} 16$ Pratiques d'écriture et champs professionnels (3) Petites fabriques d'auteurs, 1995.

Cotte, D., "Les Tic et la médiatisation des espaces de travail», Colloque "L'information dans les organisations, dynamique et complexité », Tours, mars 2006.

De Crescenzo, J.Cl, Les réseaux clandestins de salariés. Etude d'une forme de réponse organisationnelle face aux régimes managériaux de mobilisation de la force de travail. Thèse, Université Lille 3, 2005.

Delcambre, P., Ecriture et communications de travail. Pratiques d'écriture des éducateurs spécialisés, Lille, Presses Universitaires du Septentrion, 1997.

Delcambre, P., «Un service de communication en entreprise et ses histoires : le cas des chantiers de Dunkerque » in Le Moënne, Ch., Communications d'entreprises et d'organisations, Rennes, Presses Universitaires de Rennes, 1998, p.113-144.

Delcambre, P., " Quels corpus pour la recherche sur les pratiques de communication dans les entreprises et les organisations? », Sciences de la Société «La communication organisationnelle en débat », $\mathrm{n}^{\circ 50}$ 51, 2000, p.69-86.

Delcambre, P., «Un salarié a-t-il un «rapport à l'écriture»? Quelques réflexions sur une activité des officiers de la marine marchande : remplir un journal de passerelle », Pratiques $n^{\circ} 113-114$ « Images du scripteur et rapports à l'écriture », juin 2002.

Dubar, Cl., La socialisation. Construction des identités sociales et professionnelles, Paris, A. Colin, 1991.

Eraly, A., L'expression et la représentation. Une théorie sociale de la communication, Paris, L'Harmattan, 2000.

Fabre, D., (Dir.), Ecritures ordinaires, Paris, P.O.L, 1993.

Fauré, B., Les activités de production de l'information budgétaire: communications organisationnelles et régulations. Le cas d'une entreprise de BTP, Thèse Toulouse II Le Mirail, 2006.

Flahaut, Fr., La parole intermédiaire, Paris, Seuil, 1978

Fraenkel, B., La Signature, genèse d'un signe, Paris, Gallimard, 1992. 
Dossier : Migrations conceptuelles. D'où viennent les concepts de la communication organisationnelle?

Fraenkel, B., «Ecriture et performativité», Etudes de Communication $n^{\circ} 29$ «Performativité : relectures et usages d'une notion frontière », Université Lille 3, 2007.

Gaglio, G., Marcoccia, M., Zacklad, M., «L'usage des diapositives numériques en milieu organisé ou la recomposition provisoire de collectifs éclatés", Actes du Colloque SIC de Rennes, CERSIC/ERELLIF, sept 2006, p.122-126.

Goody, J., La logique de l'écriture, Paris, A. Colin, 1986.

Grosjean, M., Lacoste, M., Communication et intelligence collective. Le travail à l'hôpital, Paris, PUF, 1999.

Guilhaume, G., Les dispositifs de coaching et de formation expérientielle destinés au manager-communicateur, Thèse Université Paris 13, 2006.

Guilhaumou, J., L'avènement des porte-parole de la République (1789-1792), Essai de synthèse sur les langages de la Révolution française, Lille, Presses Universitaires du Septentrion, 1998.

Habermas, J., Théorie de l'agir communicationnel, T1 Rationalité de l'agir et rationalisation de la société, Paris, Fayard, 1987

Huët, R., Delcambre, $\mathbf{P}$, «La recherche sur corpus comme intervention visible dans le jeu social », Journées d'étude Org\&Co, " La recherche-action en communication organisationnelle », CELSA, janvier 2007.

Ion, J., (Dir.), L'engagement au pluriel, P.U. Saint-Etienne, 2001.

Jakobson, R, Essais de Linguistique générale, (Paris, Minuit, 1963), Paris, Seuil, 1970.

Joseph, I., Erving Goffman et la microsociologie, Paris, PUF, 1998

Laville, J.L., Sainsaulieu, R., Sociologie de l'association, Paris, Desclée de Brouwer, 1997.

Le Goff, J-P., Le mythe de l'entreprise, Paris, La Découverte, 1993.

Licoppe, C., «Ouvrir, suspendre et lever une audience à distance tenue par visio-conférence. Effets performatifs des actes de langage et situations équipées ", Etudes de Communication $n^{\circ} 29$ «Performativité : relectures et usages d'une notion frontière», Université Lille 3, 2007.

Mothé-Gautrat, D., Pour une nouvelle culture d'entreprise, Paris, La Découverte, 1986. 
Pour une théorie de la communication en contexte de travail...

Pène, S., « Les agencements langagiers de la qualité », in Borzeix, A., Fraenkel, B., Langage et Travail. Communication, cognition, action, CNRS Editions, 2001, p.303-321.

Pène, S., «Société de disponibilité. La vie quotidienne des communautés artificielles », HDR CELSA-Paris IV, 2005.

Rivière, C., Les rituels profanes, Paris, PUF, 1995.

Sainsaulieu, R., « La valeur travail » in Education Permanente $n^{\circ} 116$ «Comprendre le travail, ${ }^{\text {ère }}$ partie, p.159-172, 1993.

Souchier, E., Jeanneret, Y., Le Marec, J., dir, Lire, écrire, récrire. Objets, signes et pratiques des médias informatisés, BPI Ed, 2003.

Vanoye, Fr., Expression Communication, Paris, A. Colin, 1990.

Zarifian, Ph., Travail et Communication. Essai sociologique sur le travail dans la grande entreprise industrielle, Paris, PUF, 1996 